\title{
The impact of malnutrition on hospitalisation and mortality in outpatients with chronic obstructive pulmonary disease
}

\author{
P. F. Collins ${ }^{1}$, M. Elia ${ }^{1}$, T. R. Smith ${ }^{2}$, R. Kurukulaaratchy ${ }^{3}$, A. L. Cawood ${ }^{1,4}$ and R. J. Stratton ${ }^{1}$ \\ ${ }^{1}$ Institute of Human Nutrition, University of Southampton, Southampton, UK, ${ }^{2}$ Gastroenterology, Royal Bournemouth \\ Hospital, BH7 7DW, UK, ${ }^{3}$ Respiratory Medicine, Southampton University Hospital NHS Trust, Southampton SO16 6YD, UK \\ and ${ }^{4}$ Medical Affairs, Nutricia, Wiltshire BA14 OXQ, UK
}

Chronic obstructive pulmonary disease (COPD) is both a common and costly condition to the NHS ${ }^{(1)}$ and a recent survey suggests malnutrition could be present in one-fifth of outpatients with COPD ${ }^{(2)}$. This survey aimed to assess the extent to which malnutrition is related to poor clinical outcome (hospitalisation and mortality) in this patient group. Using the 'Malnutrition Universal Screening Tool', 'MUST' (3), 205 COPD outpatients (mean age 73 (SD 9.6) years; mean BMI 25.3 (SD 5.9) $\mathrm{kg} / \mathrm{m}^{2}$ ) were screened for malnutrition with subsequent healthcare use and mortality data prospectively collected 6 months post-screen.

\begin{tabular}{lcr}
\hline & \multicolumn{2}{c}{ 'MUST' } \\
\cline { 2 - 3 } & Low risk $(n=156)$ & Medium +high risk $(n=49)$ \\
\hline No. of EM and ELEC admissions per patient in 6 months & $0.65 \pm 1.1$ & $1.10 \pm 2.0$ \\
No. of EM admissions per patient in 6 months & $0.48 \pm 0.9$ & $0.92 \pm 1.8$ \\
EM and ELEC LOS per patient in 6 months (days) & $4.2 \pm 14.0$ & $6.3 \pm 14.0$ \\
EM LOS per patient in 6 months (days) & $3.3 \pm 10.4$ & $5.7 \pm 13.3$ \\
6 months mortality rate (\%) & 5.8 & $0.023^{*}$ \\
\hline
\end{tabular}

$\mathrm{EM}=$ emergency; ELEC $=$ elective; LOS = length of stay; ${ }^{*}=\chi^{2}$ test $^{\dagger}{ }^{\dagger}=\mathrm{t}$ test.

Outpatients at risk of malnutrition had almost twice the number of hospital admissions and were three-fold more likely to die within six months than those not at risk (risk ratio 2.83; $95 \%$ CI, 1.15-6.94; $P=0.023$ ) (Table 1). The impact of malnutrition on admission rate and mortality remained significant even when adjusted for age.

This survey shows that malnutrition in COPD outpatients is associated with increased hospitalisation and mortality rates.

This work was funded by an unrestricted educational grant from Nutricia.

1. Britton M (2003) Respir Med 97, S71-S79.

2. Collins PF, Stratton RJ, Kurukulaaratchy R, et al. (2009) Abstract submitted to the BAPEN Conference 2009.

3. Elia M (editor) (2003) The 'MUST' Report. Redditch, Worcs.: BAPEN (http://www.bapen.org.uk). 